\title{
CERTAIN NEAR-RINGS ARE RINGS, II
}

\author{
HOWARD E. BELL \\ Department of Mathematics \\ Brock University \\ St. Catharines, Ontario, Canada L2S $3 \mathrm{Al}$ \\ (Received August 20, 1984)
}

ABSTRACT. We investigate distributively-generated near-rings $R$ which satisfy one of the following conditions: (i) for each $x, y \in R$, there exist positive integers $m, n$ for which $x y=y^{m} x^{n}$; (ii) for each $x, y \varepsilon R$, there exists a positive integer $n$ such that $\mathrm{xy}=(\mathrm{yx})^{\mathrm{n}}$. Under appropriate additional hypotheses, we prove that $\mathrm{R}$ must be a commutative ring.

KEY WIRDS AND PHRASES. Commutativity, Distributively-generated near-rings. 1.980 AMS SUBJECT CLASSIFICATION CODE. $16 A 76$.

1. INTRODUCTION AND TERMINOLOGY.

Consider the following two properties, either of which is known to imply commutativity for rings $\mathrm{R}[3,4]$ :

$\left(C_{1}\right)$ For each $x, y \in R$, there exist positive integers $n=n(x, y)$ and $m=m(x, y)$ for which $x y=y^{m_{x}}$.

$\left(C_{2}\right)$ For each $x, y \in R$, there exists a positive integer $n=n(x, y)$ such that $\mathrm{xy}=(\mathrm{yx})^{\mathrm{n}}$.

The main purpose of this note is to show that certain distributively-generated ( $d-g)$ near-rings $R$ with these properties must be rings. The work may be regarded as a continuation of that in [2], to which paper the reader is referred for basic definitions.

Throughout the paper, the symbols $\mathrm{Z}$ and $\mathrm{Z}_{+}$will denote the integers and the positive integers respectively. The additive group of the ring $R$ will be denoted by $R^{+}$, its derived subgroup by $R^{\prime}$, and its center by $\xi(R)$. The two-sided annihilator of the subset $\mathrm{S}$ of $\mathrm{R}$ will be denoted by $\mathrm{A}(\mathrm{S})$, and the ideal generated by multiplicative commutators by $C(R)$. Such terms as center, central, commute, and commutator, unless specifically stated to refer to addition, may be assumed to refer to multiplication. The near-ring $R$ will be called strongly-distributively-generated $(s-d-g)$ if it contains a set of distributive elements whose squares generate $\mathrm{R}^{+}$.

The principal results (Theorems 2 and 4 ) are the following:

(a) Any $s-d-g$ near-ring with 1 which satisfies $\left(C_{1}\right)$ is a commutative ring.

(b) Any $d-g$ near-ring $R$ which satisfies $\left(C_{2}\right)$ and has $R^{2}=R$ is a commutative ring. 
For the proofs, we shall require the classical theorem of Fröhlich [5, p. 93] which asserts that a $\mathrm{d}-\mathrm{g}$ near-ring is distributive if and only if $\mathrm{R}^{2}$ is additively commutative. Moreover, we shall assume the easy result that in any $d-g$ near-ring $R$, the derived subgroup $\mathrm{R}^{\prime}$ of $\mathrm{R}^{+}$is an ideal.

2. A COMMUTATIVITY RESULT FOR ARBITRARY $d-g\left(C_{1}\right)$-NEAR-RINGS.

THEOREM 1. Let $R$ be any $d-g$ near-ring satisfying $\left(C_{1}\right)$. Then both $C(R)$ and $R^{\prime}$ are $n i l$ ideals. In particular, if $R$ has no non-trivial nil ideals, then $R$ is a commutative ring.

The proof follows from two lemmas.

LEMMA 1. If $R$ is any $d-g$ near-ring satisfying $\left(C_{1}\right)$, then $R$ has each of the following properties:

(a) If $a, b \varepsilon R$ and $a b=0$, then $b a=0=a x b$ for $a 11 \times \varepsilon R$.

(b) All one-sided annihilators are two-sided and are ideals of $\mathrm{R}$.

(c) Idempotent elements of $\mathrm{R}$ are central.

(d) The set $\mathrm{N}$ of nilpotent elements of $\mathrm{R}$ forms an ideal.

PROOF. Property (a) follows at once from $\left(C_{1}\right)$, and (b) follows easily from (a). To establish (c), let $e$ be an idempotent and $x \in R$. Then for some $p, q \geqslant 1, x e=e^{p} x^{q}$ $=e x^{q}$ and hence exe $=e x^{q}=x e$; similarly, ex $=x^{r} e$ for some $r \geqslant 1$, so that exe $=x^{r} e$ $=$ ex. Thus ex $=x e$.

To establish (d), it will suffice to show that $\mathrm{N}$ forms an additive subgroup [2, Lemma 1]; and this may be done by proving that for each $a, b \in N$ and each positive integer $j,(a-b)^{j}$ is a finite sum $\Sigma \pm p_{i}$, where each $p_{i}$ is a finite product of elements of $R$, of which at least $j$ belong to $T=\{a, b\}$. To see that this is enough, note that if $a^{n}=b^{m}=0$ and if $j=n+m-1$, then each of the summands $p_{i}$ will be zero by (a).

We proceed by induction on $j$, the case $j=1$ being trivial. Suppose the result holds for $j$ and write

$$
(a-b)^{j+l}=(a-b)(a-b)^{j}=(a-b)\left(\Sigma \pm p_{i}\right)=\Sigma \pm(a-b) p_{i} \text {, }
$$

where each $p_{i}$ is a product having at least $j$ factors from $T$; and for each $p=p_{i}$ write $(a-b) p=p^{k}(a-b)^{m}=\left(p^{k} a-p^{k} b\right)(a-b)^{m-1}$. If $m=1$, we are finished; otherwise, express $(a-b)^{m-1}$ in the form $\Sigma \pm d_{s}$ where the $d_{s}$ are distributive. Since $(a-b) p=\Sigma \pm\left(p k_{a d}-p^{k_{b d}}\right)$ and since each of the products $\mathrm{p}^{\mathrm{k}_{\mathrm{ad}}}$ and $\mathrm{p}^{\mathrm{k}_{\mathrm{bd}}}$ has at least $j+1$ factors from $T$, the inductive step, and hence the proof of (d), is complete.

In view of $(d)$, the proof of Theorem 1 will be complete once we establish the following lemma.

LEMMA 2. Let $R$ be a d-g near-ring satisfying $\left(C_{1}\right)$ and having no non-zero nilpotent elements. Then $R$ is a commutative ring.

PROOF. By Lemma 3 of $[1], R$ is a subdirect product of homomorphic images having no zero divisors; thus we assume that $R$ has no zero divisors. Note that if $R$ is multiplicatively commutative, hence distributive, then $\mathrm{R}^{2}$ is additively commutative; therefore, for $a 11 a, b \varepsilon R$

$$
0=a^{2}+a b-a^{2}-a b=a(a+b-a-b)=a+b-a-b,
$$

so that $\mathrm{R}^{+}$is abelian. Observe also that if $\mathrm{e}$ is any non-zero idempotent, the fact that $e(x-e x)=(x-x e) e=0$ for all $x \varepsilon R$ shows that $e$ must be a multiplicative identity element. 
Assume, then, that $\mathrm{R}^{+}$is not abelian; hence $\mathrm{R}$ is not commutative. Let $a$ and $b$ be elements of $R$ which do not commute; and let $\mathrm{m}, \mathrm{n}, \mathrm{s}, \mathrm{t}$ be positive integers, at least one of which is greater than 1 , for which $a b=b^{m} a^{n}=a^{n s} b^{m t}$. If $n s=1$, then $a\left(b-b^{m t}\right)=0$, and hence $b^{m t-1}$ is a non-zero idempotent. If $n s>1$, then $a b^{\cdot}=a^{n s-1} a b^{m t}=\left(a b^{m t}\right)^{v}$ $\left(a^{n s-l}\right)^{w}$ for appropriate positive integers $v$, w; and there exists an element $c$, which is either $a$ or an element of the form ya, such that $a b=a b c$. If follows that $c$ is idempotent, and incidentally that $c$ must have been of the form ya.

So far we have shown that any non-central element a of $R$ either has a left inverse or has the property that for any $b$ not commuting with $a, b^{k}=1$ for some positive integer $k$. Suppose the latter holds, let $c_{1}$ be an element not commuting with a, and use $\left(C_{1}\right)$ to obtain $c$ for which $a c_{1}=c a$. It is easily verified that ca does not commute with a, so $(c a)^{k}=1$ for some integer $k$; and in this case also, a has a left inverse. Now suppose that $z$ is any non-zero central element, and that a is non-central. Then $a z=z a$ is also non-central, so az has a left inverse, and therefore $z$ has a left inverse. Thus, $R$ is a division near-ring; and since division near-rings have commutative addition, we have contradicted our initial assumption concerning $R$. Hence, $R^{+}$is abelian and $R$ is a ring. Multiplicative commutativity follows from the result of [3].

3. COMMUTATIVITY OF s-d-g $\left(C_{1}\right)$-NEAR-RINGS WITH 1.

The major theorem of this section is the following:

THEOREM 2. If $R$ is a strongly-distributively-generated near-ring with 1 which has property $\left(\mathrm{C}_{1}\right)$, then $\mathrm{R}$ must be a commutative ring.

of course, it suffices to prove the theorem under the additional assumption that $\mathrm{R}$ is subdirectly irreducible $(s-i)$. The lemmas which follow all treat the subdirectly irreducible case.

LEMMA 3. Let $\mathrm{R}$ be a s-i $\mathrm{d}-\mathrm{g}$ near-ring with 1 , in which all idempotents are central. Then 1 is the only non-zero idempotent.

PROOF. If $e$ is any non-zero idempotent, the centrality of e enables us to show that 1-e is idempotent as wel1. Clearly $\operatorname{Re} \subseteq \mathrm{A}(1-\mathrm{e})$; moreover, if $\times \varepsilon \mathrm{A}(1-\mathrm{e})$, the representation $x=x(1-e)+x e$ shows that $x=x e \varepsilon$ Re. Thus, $R e=A(1-e)$, and similarly $R(1-e)=A(e)$. But it is easy to show that $A(x)$ is an ideal for any central $x$, so Re and $\mathrm{R}(1-\mathrm{e})$ are ideals, which obviously have trivial intersection. The subdirect irreducibility of $R$ therefore forces $R(1-e)$ to be trivial, so that $e=1$.

LEMMA 4. Let $R$ be a s-i d-g $\left(C_{1}\right)$-near-ring with 1 . Then

(a) if $\mathrm{x} \varepsilon \mathrm{R}$, either $\mathrm{x}$ commutes with -1 or there exists $\mathrm{k} \varepsilon \mathrm{Z}_{+}$such that $\mathrm{x}^{\mathrm{k}}=1$;

(b) for each $x \in R, x^{2}(-1)=(-1) x^{2}$.

PROOF. (a) Suppose $x(-1) \neq(-1) x$, and chose $k, j, m, n \varepsilon Z_{+}$such that $x(-1)=(-1)^{k} x^{j}=x^{j n}(-1)^{k m}$.

Assume first that $j n>1$. If $k m$ is odd, we have $x=x^{j n}$, which implies that $x^{j n-1}$ is a non-zero idempotent, necessarily equal to 1 . If $\mathrm{km}$ is even, (3.1) yields $-x=x^{j n}$, hence $x=-x^{j n}=x^{j n-1} x(-1)=x^{j n-1} x^{j n}$ and $x^{2(j n-1)}=1$.

On the other hand, if $j n=1$, then (3.1) yields $-x=x(-1)=x$. Choose $q$, s $\varepsilon Z_{+}$ with $(-1) x=x^{s}(-1)^{q}$. Since $x(-1)=x$, this implies $(-1) x=x^{s}$ with $s>1$; and we conclude that $x=(-1) x^{s}=((-1) x) x^{s-1}=x^{2 s-1}$, so that $x^{2(s-1)}=1$. 
(b) It follows from part (a) that zero divisors commute with -1 , hence we may assume $x$ is not a zero divisor. Now if $x(-1)=x^{s}$ for some $s \in Z_{+}$, $x$ commutes with $x(-1)$ and consequently $x(-1)=(-1) x$; therefore, we may assume that $x(-1)=(-1) x^{s}$ for some $s \in Z_{+}$. Thus, $(-1) \times(-1)=x^{s}$; and commuting $x$ with $(-1) \times(-1)$ gives $x(-1) \times(-1)=$ $(-1) x(-1) x$ or $(-x)^{2}=(-1)(-x)(x)$. In this equality we may replace $x$ by $-x$, since $x$ commutes with -1 if and only if $-x$ does; therefore $x^{2}=(-1) x(-x)$ and $(-1) x^{2}=x(-x)=-x^{2}=x^{2}(-1)$.

LEMMA 5. Let $R$ be a $s-i d-g\left(C_{1}\right)$-near-ring with 1 , and let $D$ be the set of zero divisors. Then

(a) $\mathrm{D}$ is an ideal and $\mathrm{D}^{+}$is abelian;

(b) $R^{\prime}=C(R) \subseteq A(D)$;

(c) if $d \varepsilon D$ and $x$ does not commute with $d$, then there exists $s \varepsilon Z_{+}$such that $x d=d x^{s}$.

PROOF. (a) Let $\mathrm{S}$ be the heart of $\mathrm{R}$ - that is, the intersection of all non-zero ideals; to show $D$ is an ideal, we show that $D=A(S)$. Clearly $A(S) \subseteq D$; conversely, if $d \in D, A(d)$ is a non-trivial ideal, hence $S \subseteq A(d)$ and $d \in A(S)$. Therefore $D=A(S)$. Note that by Lemma $4(a)$, all elements of $D$ commute with -1 ; thus if $d_{1}, d_{2} E D$, we have $-\mathrm{d}_{1}-\mathrm{d}_{2}=(-1)\left(\mathrm{d}_{1}+\mathrm{d}_{2}\right)=\left(\mathrm{d}_{1}+\mathrm{d}_{2}\right)(-1)=-\mathrm{d}_{2}-\mathrm{d}_{1}$, so $\mathrm{D}^{+}$is abelian.

(b) If $x, y \in R$ and $d \varepsilon D$, then $d x$ and $d y$ are in $D$; hence $d x+d y-d x-d y=0=$ $\mathrm{d}(\mathrm{x}+\mathrm{y}-\mathrm{x}-\mathrm{y})$, and $\mathrm{R}^{\prime} \subseteq \mathrm{A}(\mathrm{D})$. By Fröhlich's theorem and the commutativity of rings satisfying $\left(C_{1}\right)$, a $d-g\left(C_{1}\right)$-near-ring with 1 is additively commutative if and only if it is multiplicatively commutative; and applying this observation to $R / C(R)$ and $R / R^{\prime} g i v e s$ $C(R)=R^{\prime}$.

(c) By part (b) we have $d(d y-y d)=0-$ that is, $d^{2} y=d y d-$ for all y $\varepsilon$; moreover, since $y d=d y_{1}$ for some $y_{1} \varepsilon R$, we get $y^{2}=d y_{1} d=d^{2} y_{1}=d y d$ as well. Thus, for all $d \varepsilon D$, all y $\varepsilon R$ and all integers $i \geqslant 2$, we have $d^{i} y=y d^{i}$. Suppose that $d \varepsilon D$ and $x \in R$ are such that $x d=d^{m} x^{s}$ for some $m>1$, and choose $n, q$ such that $d x=x^{n_{d}} q$. Then $x d=d^{m} x^{s}=d^{m-1}(d x) x^{s-1}=d^{m-1} x^{n} d^{q} x^{s-1}=d^{m+q-1} x^{n+s-1}$, and $d x=x^{n} d^{q}=x^{n-1}(x d) d^{q-1}$ $=x^{n-1} d^{m} x^{s} d^{q-1}=x^{n+s-1} d^{m+q-1}$. Since $m+q-1>1$, we thus have $x d=d x$, so our proof is finished.

LEMMA 6. Let $R$ be $s-i$ and $d-g$ with 1 , and suppose $R$ satisfies $\left(C_{1}\right)$. Then $2 R^{\prime}=0$.

PROOF. By Theorem $1, R^{\prime} \subseteq D$. By Lemma 4(a), all elements of $R^{\prime}$ commute with -1 ; in particular, for all $\mathrm{x}, \mathrm{y} \varepsilon \mathrm{R}, \mathrm{x}+\mathrm{y}-\mathrm{x}-\mathrm{y}+\mathrm{y}-\mathrm{x}-\mathrm{y}+\mathrm{x}=\mathrm{x}+\mathrm{y}-\mathrm{x}-\mathrm{x}-\mathrm{y}+\mathrm{x}$ commutes with -1 . Taking for $x$ and $y$ a pair $r, s$ of distributive elements and simplifying, we get the result that $2(r+s-r-s)=0$, from which it follows that $2 R^{\prime}=0$. LEMMA 7. For $R$ a s-i d-g $\left(C_{1}\right)$-near-ring with $1, A(2) \subseteq \xi(R)$. In particular, $R^{\prime}$ $\subseteq \xi(R)$ and therefore, $2 R \subseteq \xi(R)$.

PROOF. If $2 \xi \mathrm{D}, \mathrm{R}^{+}$is abelian by Lemma 6 ; hence assume $2 \varepsilon \mathrm{D}$. Let $\mathrm{x} \in \mathrm{A}(2)$; and by Lemma $5(c)$, choose $k \varepsilon Z_{+}$such that $(1+x) 2=2(1+x)^{k}=2$. Thus, $1+x+1+x=1+1$, which yields $x+1=1+x$. If $b^{k}=1$ for some $k \varepsilon z_{+}$, we now get $b+x-b-x=b\left(1+b^{k-1} x-1-b^{k-1} x\right)=0$. By Lemma 4(a), the only elements $b$ yet to be considered commute with -1 ; and since $x$ commutes additively with $b$ if and only if it commutes additively with $b+x$, we may assume $b+x$ commutes with -1 also. Since $x \in D, x$ commutes with -1 , and it follows at once that $b+x=x+b$. Thus, $A(2) \subseteq \xi(R)$. 
That $R^{\prime} \sqsubseteq \xi(R)$ is now clear from Lemma 6. Noting that $2 R=R 2$, we complete the proof of the lemma by showing that $x+x+y=y+x+x$ for all $x, y \in R$. But since $y-x=-x+y+c$ for some $c \varepsilon R^{\prime}$ and since $R^{\prime} \cong \xi(R)$, we have $x+x+y-x-x-y=$ $x+y+c-x-y=x+y-x-y+c=y+c-y+c=c+c=0$, the last equality following from Lemma 6 .

PROOF OF THEOREM 2. We need only show that $\mathrm{R}^{+}$is abelian, since the theorem then follows from Fröhlich's theorem and the theorem of [3]. Let $r$, s be arbitrary distributive elements of $R$. By Lemma $4(b),(r+s)^{2}$ commutes with -1 , which means that

$$
-r^{2}-s r-r s-s^{2}=-s^{2}-r s-s r-r^{2} \text {. }
$$

If we write $r s=c+s r$, where $c=r s-s r \varepsilon \xi(R)$, and recall that $2 s r \varepsilon \xi(R)$, we can write $(3.2)$ as

$$
-r^{2}-s^{2}-2 s r-c=-s^{2}-r^{2}-2 s r-c .
$$

It follows at once that $r^{2}+s^{2}=s^{2}+r^{2}$, and the fact that $R$ is strongly-distributivelygenerated implies that $\mathrm{R}^{+}$is abelian.

I conjecture that Theorem 2 remains true if $R$ is merely assumed to be $d-g$ rather than $s-d-g$, but a proof eludes me. However, all the machinery is in place to establish two interesting cases of the conjecture.

THEOREM 3. Let $R$ be a $d-g$ near-ring with 1 , and suppose $R$ satisfies one of the following specialized versions of $\left(C_{1}\right)$ :

$\left(C_{3}\right)$ For each $x, y \in R$, there exists an integer $n=n(x, y) \geqslant 1$ for which $x y=y x^{n}$.

$\left(C_{4}\right)$ For each $x, y \varepsilon R$, either $x y=y x$ or there exist $m, n \varepsilon z_{+}$with $m>2$, such that $x y=y^{m} x^{n}$.

Then $\mathrm{R}$ is a commutative ring.

PROOF. Again we may assume that $R$ is subdirectly irreducible and (by Lemma 6) that $2 \in D$. Arguments similar to that of Lemma 5(c) show that zero divisors are central, and commuting 2 with $\mathrm{r}+\mathrm{s}$ for arbitrary distributive $\mathrm{r}$, $s$ now shows $\mathrm{R}^{+}$is abelian.

4. COMMUTATIVITY OF $\mathrm{d}-\mathrm{g}\left(\mathrm{C}_{2}\right)$-NEAR-RINGS.

THEOREM 4. If $R$ is any $d-g$ near-ring satisfying $\left(C_{2}\right)$, then $R$ is commutative. Moreover, if $R^{2}=R$, then $R$ is a ring.

PROOF. Note first that idempotents are central, for if $e$ is idempotent and $x \in R$, and if we choose $n, m \varepsilon Z_{+}$such that $e x=(x e)^{n}$ and $x e=(e x)^{m}$, right-multiplying the first of these equalities by $e$ and left-multiplying the second by $e$ yields ex $=$ exe $=x e$.

Now if $R$ is any $d-g\left(C_{2}\right)$-near-ring and $a, b \in R$ are such that $a b \neq b a$, there exist $\mathrm{m}, \mathrm{n}>\mathrm{l}$ such that

$$
a b=(b a)^{n} \text { and } b a=(a b)^{m} ;
$$

it follows that

$$
\mathrm{ab}=(\mathrm{ab})^{\mathrm{nm}} \text { and } \mathrm{ba}=(\mathrm{ba})^{\mathrm{nm}},
$$

and hence that $(a b)^{n m-1}$ and $(b a)^{n m-1}$ are both idempotent. In fact, if $(a b)^{t}=e$ is idempotent, $(4.1)$ shows that $(b a)^{t}=(a b)^{m t}=e$, hence $(a b)^{n m-1}$ and (ba) $)^{n-1}$ are equal 
to the same idempotent, say $e_{1}$.

We treat first the case of $R$ with 1 , and as usual consider the subdirectly irreducible case. If we suppose $R$ contains a pair of non-commuting elements $a, b$ and choose $n$, $m$ as above, then Lemma 3 guarantees that $(a b)^{n m-1}=(b a)^{n m-1}=1$, so that a and $b$ are both invertible. We choose $q \varepsilon z_{+}$such that $\left(b^{-1} a\right) b=\left(b\left(b^{-1} a\right)\right)^{q}$, which reduces at once to $a b=b a^{q}$. Thus, $R$ is a commutative ring by Theorem 3 .

We now drop the hypothesis that $R$ has 1 . Again suppose $a b \neq$ ba and let $n, m$ and $e_{1}$ be as above. The near-ring $e_{1} R$ is a $d-g\left(C_{2}\right)$-near-ring having $e_{1}$ as multiplicative identity, hence is commutative. Therefore $e_{1} a_{1} b-e_{1} b_{1} a=0=e_{1}(a b-b a)$; and since $e_{1}$ $=(a b)^{n m-1}=(b a)^{n m-1}$, an appeal to (4.2) yields the contradiction $a b=b a$. Hence $R$ must be commutative; and if $R^{2}=R$, Fröhlich's theorem shows that $R$ is a ring.

ACKNOWLEDGEMENT:

Supported by the Natural Sciences and Engineering Research Council of Canada, Grant No.

A 3961 .

\section{REFERENCES}

1. BELL, H. E. Near-rings in which each element is a power of itself, Bull. Austral. Math. Soc. 2 (1970), 363-368.

2. BELL, H. E. Certain near-rings are rings, J. London Math. Soc. (2) 4(1971), $264-270$.

3. BELL, H. E. A commutativity condition for rings, Canad. J. Math. 28 (1976), 986-991.

4. CHACRON, M. and THIERRIN, G., o-reflexive semigroups and rings, Canad. Math. Bull. 15 (1972), 185-188.

5. FRüHLICH, A. Distributively-generated near-rings, I, Ideal theory, Proc. London Math. Soc. (3) $8(1958), 76-94$. 


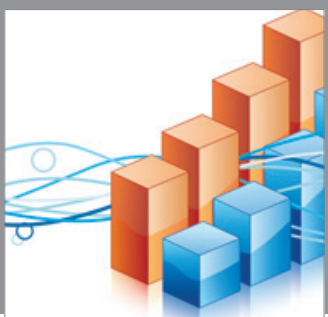

Advances in

Operations Research

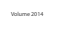

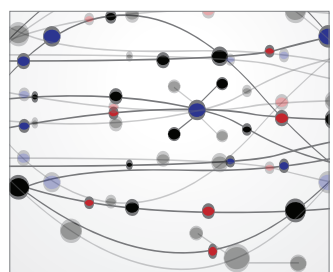

\section{The Scientific} World Journal
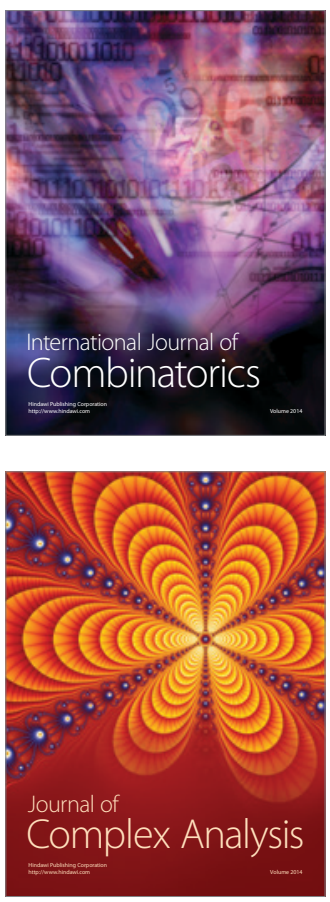

International Journal of

Mathematics and

Mathematical

Sciences
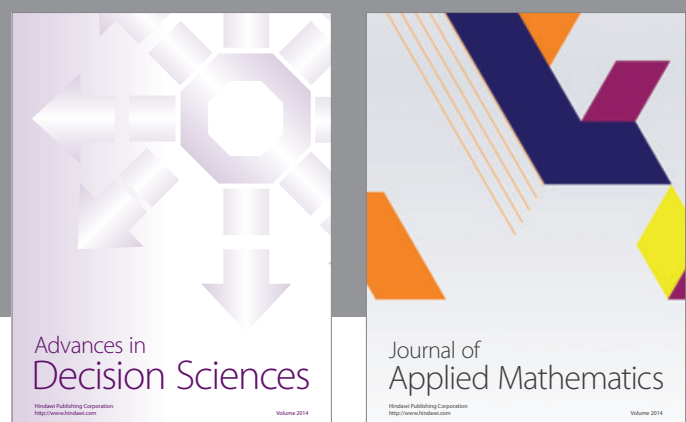

Journal of

Applied Mathematics
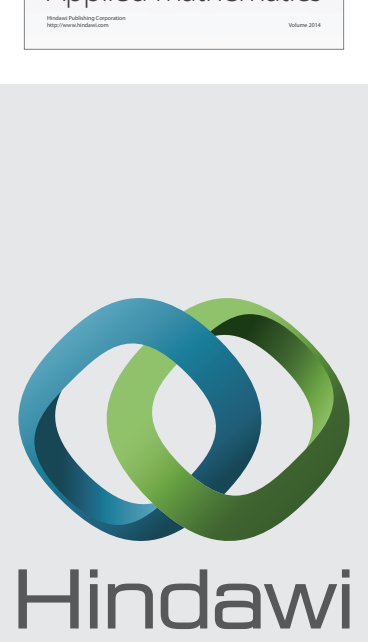

Submit your manuscripts at http://www.hindawi.com
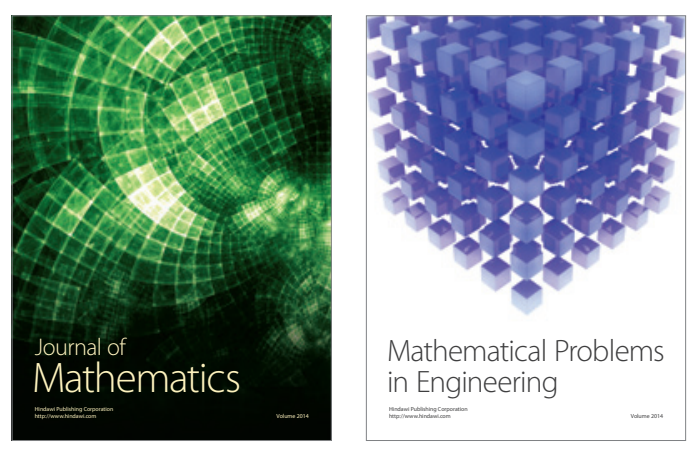

Mathematical Problems in Engineering
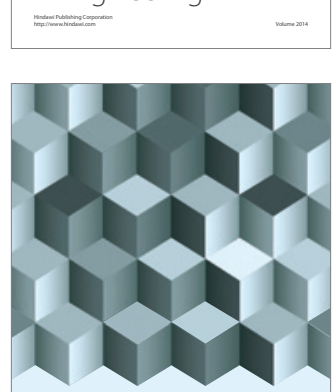

Journal of

Function Spaces
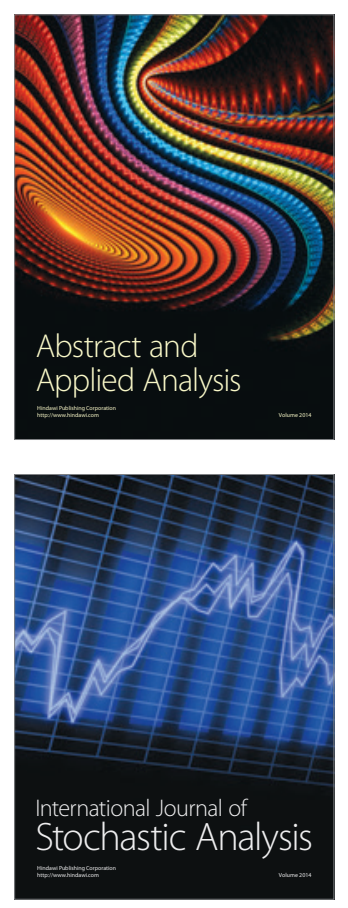

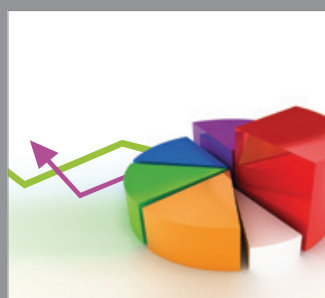

ournal of

Probability and Statistics

Promensencen
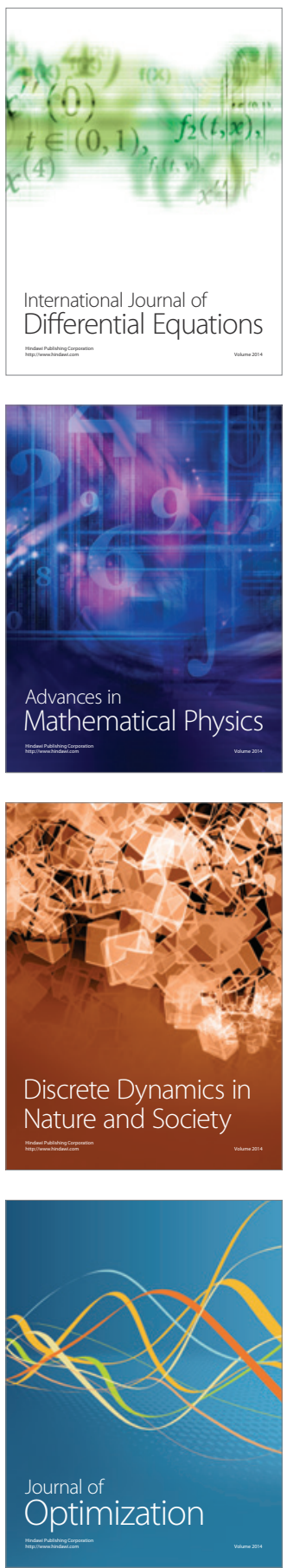